\title{
Quasi-Dirac neutrino oscillations at DUNE and JUNO
}

\author{
G. Anamiati, ${ }^{1, *}$ V. De Romeri, ${ }^{1, \dagger}$ M. Hirsch, ${ }^{1, *}$ C. A. Ternes, ${ }^{1, \S}$ and M. Tórtola ${ }^{1,2, \|}$ \\ ${ }^{1}$ Instituto de Física Corpuscular, CSIC-Universitat de València, 46980 Paterna, Spain \\ ${ }^{2}$ Departament de Física Teòrica, Universitat de València, 46100 Burjassot, Spain
}

(Received 10 July 2019; published 28 August 2019)

\begin{abstract}
Quasi-Dirac neutrinos are obtained when the Lagrangian density of a neutrino mass model contains both Dirac and Majorana mass terms, and the Majorana terms are sufficiently small. This type of neutrino introduces new mixing angles and mass splittings into the Hamiltonian, which will modify the standard neutrino oscillation probabilities. In this paper, we focus on the case where the new mass splittings are too small to be measured, but new angles and phases are present. We perform a sensitivity study for this scenario for the upcoming experiments DUNE and JUNO, finding that they will improve current bounds on the relevant parameters. Finally, we also explore the discovery potential of both experiments, assuming that neutrinos are indeed quasi-Dirac particles.
\end{abstract}

DOI: $10.1103 /$ PhysRevD.100.035032

\section{INTRODUCTION}

Since the discovery of solar and atmospheric neutrino oscillations about two decades ago, neutrino oscillation experiments have become more and more sophisticated. Nowadays many of the parameters characterizing the conversion of neutrino flavors in the standard 3-neutrino picture are rather well measured [1]. However, this framework might not be complete and might need to be extended. Several studies considering global oscillation data have been performed assuming the existence of new physics beyond the standard sector; see e.g., Refs. [2-4]. One of these scenarios, which will be considered here, is the case of quasi-Dirac neutrinos [5].

Since neutrino oscillations are blind to the Dirac or Majorana nature of neutrinos, one needs other types of experiments, e.g., those searching for neutrinoless double beta decay to determine it [6,7]. In general, one can say that the Dirac case, consisting of $n$ neutrinos, is a limiting case of the more general Majorana scenario, with $2 n$ neutrinos. This limit is performed by putting the Majorana mass terms in the Lagrangian to zero. Quasi-Dirac neutrinos arise from the presence of both Majorana and Dirac mass terms in the Lagrangian simultaneously, where the Majorana terms are

\footnotetext{
*anamiati@ific.uv.es

deromeri@ific.uv.es

¥mahirsch@ific.uv.es

\$chternes@ific.uv.es

"mariam@ific.uv.es
}

Published by the American Physical Society under the terms of the Creative Commons Attribution 4.0 International license. Further distribution of this work must maintain attribution to the author(s) and the published article's title, journal citation, and DOI. Funded by SCOAP ${ }^{3}$. small but not exactly zero. As we will show, the departure from Diracness - i.e., nonzero Majorana mass terms-leads to the presence of new mixing angles and new mass splittings, which will affect neutrino oscillation probabilities.

Along this work, we will use "quasi-Dirac neutrinos" to refer to active-sterile neutrino pairs [8]. In order to distinguish this scenario from the one with active-active pairs, we denote the latter ones as pseudo-Dirac neutrinos [9]. Many aspects of pseudo-Dirac neutrinos have been studied in the literature; see e.g., Refs. [10-17]. Note, however, that models with pseudo-Dirac neutrinos do not fit oscillation data anymore [18-20]. In the context of quasi-Dirac neutrinos, many papers appeared in the literature proposing explanations for the solar and atmospheric neutrino problems [21-23], as well as consistent descriptions of standard and short baseline neutrino oscillations $[24,25]$. Several papers derived limits on quasi-Dirac neutrino properties from different data sets [26,27], while others discussed them in the context of neutrino telescopes [28-31].

From a theoretical point of view, there are several options on how quasi-Dirac neutrinos can be created. They can be produced, for instance, in models with a singular seesaw [32,33], double seesaw [34] or Dirac-seesaw [35] mechanisms. Another possibility is to obtain them from extended gauge groups [36,37] or even in supergravity theories [38].

Because of the presence of new spinors in Dirac neutrino models, there is some overlap between the study of quasiDirac neutrinos and the scenario with sterile neutrinos. Several experimental hints point towards the existence of sterile neutrinos, which have been extensively investigated in many experiments. The possible observation of short baseline oscillations in some of these experiments [39-46], together with the nonobservation of neutrino oscillations in 
others [47-56], leads to large tensions in the global $3+1$ picture $[2,3,57,58]$, which cannot be reconciled even by adding more than one sterile neutrino [59]. For a recent review on this topic, we refer the reader to Refs. $[60,61]$. Even though there is some theoretical overlap, these results would point towards new mass splittings at the $\sim 1 \mathrm{eV}^{2}$ scale. Therefore, this type of oscillation cannot be explained with quasi-Dirac neutrinos, whose additional mass splittings are constrained to be much below the eV scale.

In this paper, we study the sensitivity of the upcoming Deep Underground Neutrino Experiment (DUNE) [62-64] and Jiangmen Underground Neutrino Observatory (JUNO) [65] to quasi-Dirac neutrino oscillations. The DUNE experiment, hosted by Fermilab, will exploit the synergy of a very high intense neutrino beam and two massive argon detectors to carry on a broad research program in neutrino physics. DUNE will allow us to perform tests of the threeneutrino paradigm with remarkable sensitivity, in particular, concerning neutrino oscillation parameters [66-68]. The high intensity of the neutrino beam as well as the high resolution of the near and far detectors, which characterize DUNE, will make it a leading experiment also in the search for new physics. Hence, besides pursuing a comprehensive study of the neutrino mixing, DUNE will also allow us to explore new physics scenarios, for instance, via the search for nonstandard interactions [69-71] or sterile neutrinos [72,73], among others [74-77]. JUNO is a next-generation reactor experiment and will be located $53 \mathrm{~km}$ from the Yangjiang (six cores with $2.9 \mathrm{GW}_{\text {th }}$ thermal power each) and Taishan (four cores with $4.6 \mathrm{GW}_{\text {th }}$ thermal power each) nuclear power plants. The current Daya Bay complex will also contribute roughly $3 \%$ to the total antineutrino flux. The JUNO detector will be made of 20 kton of liquid scintillator. With these powerful sources and an excellent energy resolution, JUNO will be expecting around $10^{5}$ inverse beta decay events in total. Huge statistics and the long baseline (for a reactor experiment) assure a measurement of $\sin ^{2} \theta_{12}, \Delta m_{21}^{2}$ and $\Delta m_{e e}^{2}[78,79]$ at below $1 \%$ level, which makes it a very complementary experiment to DUNE. Our paper is structured as follows. In Sec. II we present the theoretical framework for quasi-Dirac neutrinos. The simulation of the DUNE and JUNO experiments is described in Sec. III. Next, we discuss our results in Sec. IV and finally draw our conclusions in Sec. V.

\section{QUASI-DIRAC NEUTRINO OSCILLATIONS}

A pair of quasi-Dirac neutrinos is a pair of Majorana neutrinos with a small mass splitting and a relative $C P$ sign between the two states. For the sake of illustration, let us start by considering only one neutrino generation. In this case, in the basis $\left(\nu, N^{c}\right)$, where $\nu$ and $N^{c}$ are the active and the sterile neutrinos, respectively, the most general neutrino mass matrix is

$$
m_{\nu}=\left(\begin{array}{cc}
m_{L} & m_{D} \\
m_{D} & m_{R}
\end{array}\right)
$$

Here, $m_{L}$ and $m_{R}$ are the terms that violate lepton number, while $m_{D}$ is the standard Dirac neutrino mass term. In the limit in which $m_{L}$ and $m_{R}$ are equal to zero, lepton number is conserved and neutrinos are Dirac particles. This limiting case is characterized by two degenerate mass eigenstates

$$
\begin{aligned}
& \nu_{1}=\frac{1}{\sqrt{2}}\left(\nu+N^{c}\right), \\
& \nu_{2}=\frac{i}{\sqrt{2}}\left(-\nu+N^{c}\right),
\end{aligned}
$$

where the factor $i$ is introduced such that both mass eigenvalues are positive. Note that, in this mass eigenstate basis, both $\nu_{1}$ and $\nu_{2}$ are equal mixtures of active and sterile neutrinos. Small deviations from the limit $m_{L}=m_{R}=0$ then lead to quasi-Dirac neutrinos. If we define the new variables $\varepsilon=\left(m_{L}+m_{R}\right) /\left(2 m_{D}\right)$ and $\theta=\left(m_{L}-m_{R}\right) /\left(4 m_{D}\right)$, in the limit $\varepsilon, \theta \ll 1$, one can rewrite Eq. (2) as

$$
\begin{aligned}
& \nu_{1} \simeq \frac{1}{\sqrt{2}}\left[(1+\theta) \nu+(1-\theta) N^{c}\right], \\
& \nu_{2} \simeq \frac{i}{\sqrt{2}}\left[(-1+\theta) \nu+(1+\theta) N^{c}\right],
\end{aligned}
$$

where the quasidegenerate pairs are nearly maximally mixed and $\theta$ is a small angle describing the departure from maximality. The masses are given by

$$
m_{1,2} \simeq m_{D}(1 \pm \varepsilon) .
$$

Quasi-Dirac neutrinos are therefore characterized by new mass splittings and new mixing angles.

Let us now consider the extension of the standard model (SM) with three sterile neutrinos $N^{c}$. In the physical mass eigenstate basis, the charged current SM Lagrangian is modified to

$$
\mathcal{L}_{C C}=-\frac{g}{\sqrt{2}} W_{\mu}^{-} \sum_{l=1}^{3} \sum_{j=1}^{6} \mathbf{V}_{l j} \bar{\ell}_{l} \gamma^{\mu} P_{L} \nu_{j}+\text { H.c. },
$$

where $P_{L, R}=\left(1 \mp \gamma_{5}\right) / 2$ are the chirality projectors, $l=1,2,3$ denote the flavor of the charged leptons, and $j=1, \ldots, 6$ are the physical neutrino states. The mixing is parametrized by a rectangular $3 \times 6$ mixing matrix, $\mathbf{V}_{l j}$ [80]. Moreover, the addition of the three sterile neutrinos allows for the mass term

$$
\mathcal{L}_{\text {mass }}=\frac{1}{2} \bar{\nu}_{\alpha} M_{\alpha \beta} \nu_{\beta}+\text { H.c. }
$$


Here, indices $\alpha, \beta=1,2,3(4,5,6)$ are for active (sterile) neutrinos, and $M_{\alpha \beta}$ is the generalization of Eq. (1) for three generations. The full neutrino mass matrix is now diagonalized by a $6 \times 6$ unitary matrix, $\tilde{\mathbf{U}}$. We parametrize the neutrino mixing matrix as

$$
\begin{aligned}
& \tilde{\mathbf{U}}\left(\theta_{i j}, \delta_{i j}\right) \\
& =\hat{R}_{56} \hat{R}_{46} \hat{R}_{36} \hat{R}_{26} \hat{R}_{16} \hat{R}_{45} \hat{R}_{35} \hat{R}_{25} \hat{R}_{15} \hat{R}_{34} \hat{R}_{24} \hat{R}_{14} \hat{R}_{23} \hat{R}_{13} \hat{R}_{12},
\end{aligned}
$$

where $\hat{R}_{i j}$ are complex rotation matrices which depend on the mixing angles $\theta_{i j}$ and $C P$-violating phases $\delta_{i j}$. The rotation matrices $\hat{R}_{i j}$ are parametrized in the usual way. For example, for $\hat{R}_{14}$ we have

$$
\hat{R}_{14}=\left(\begin{array}{cccccc}
\cos \theta_{14} & 0 & 0 & e^{-i \delta_{41}} \sin \theta_{14} & 0 & 0 \\
0 & 1 & 0 & 0 & 0 & 0 \\
0 & 0 & 1 & 0 & 0 & 0 \\
-e^{i \delta_{41}} \sin \theta_{14} & 0 & 0 & \cos \theta_{14} & 0 & 0 \\
0 & 0 & 0 & 0 & 1 & 0 \\
0 & 0 & 0 & 0 & 0 & 1
\end{array}\right)
$$

Note that the matrix $\tilde{\mathbf{U}}$ in Eq. (6) contains the mixing among sterile neutrinos, which is not observable in neutrino oscillation experiments. Thus, we will neglect these rotations in the following. In the remaining rotations, we have, in general, 12 angles and 12 phases. However, in our numerical studies we will limit ourselves to two phases only, namely, $\delta_{13}$ and $\delta_{16}$. This means that the mixing matrix above can be reduced to

$$
\tilde{\mathbf{U}}\left(\theta_{i j}, \delta_{i j}\right)=R_{36} R_{26} \hat{R}_{16} R_{35} R_{25} R_{15} R_{34} R_{24} R_{14} R_{23} \hat{R}_{13} R_{12},
$$

where $R_{i j}$ denote real rotations. It proves convenient to multiply $\tilde{\mathbf{U}}$ by the following $6 \times 6$ rotation matrix [as in Eq. (16) of Ref. [5] ]:

$\mathbf{U}\left(\theta_{i j}, \delta_{i j}\right) \equiv \tilde{\mathbf{U}}\left(\theta_{i j}, \delta_{i j}\right) W, \quad$ with $\quad W=\frac{1}{\sqrt{2}}\left(\begin{array}{cc}I_{3} & i I_{3} \\ I_{3} & -i I_{3}\end{array}\right)$,

with $I_{3}$ being the $3 \times 3$ identity matrix. This redefinition allows us to trivially recover the Dirac limit for the mixing matrix by putting to zero all nonstandard angles. The probability of a neutrino oscillating from a flavor $\alpha$ to a flavor $\beta$ can then be written as

$$
P\left(\nu_{\alpha} \rightarrow \nu_{\beta}\right)=\left|\sum_{j=1}^{6} \mathbf{U}_{\beta j} \mathbf{U}_{\alpha j}^{*} \exp \left(-\frac{i m_{j}^{2} L}{2 E}\right)\right|^{2},
$$

where $L$ is the length traveled by the neutrino and $E$ its energy. Therefore, neutrino oscillations are described by the Hamiltonian

$$
\mathcal{H}_{0}=\frac{1}{2 E} \mathbf{U M}^{2} \mathbf{U}^{\dagger},
$$

where $\mathbb{M}^{2}=\operatorname{diag}\left(0, \Delta m_{21}^{2}, \Delta m_{31}^{2}, \epsilon_{1}^{2}, \Delta m_{21}^{2}+\epsilon_{2}^{2}, \Delta m_{31}^{2}+\epsilon_{3}^{2}\right)^{1}$ and the square of the lightest neutrino mass, $m_{1}^{2}$, has been subtracted from the diagonal elements in the $\mathbb{M}^{2}$ matrix, as usual. To include matter effects on the neutrino propagation, one should add the effective matter potential to the neutrino Hamiltonian above. Quasi-Dirac neutrinos feel the same potential in the 4-5-6 sector as in the 1-2-3 sector. Thus,

$$
\mathcal{H}=\frac{1}{2 E}\left(\mathbf{U M}^{2} \mathbf{U}^{\dagger}+\mathbb{A}\right),
$$

where the potential is now given by $\mathbb{A}=\operatorname{diag}\left(V_{\mathrm{CC}}+\right.$ $\left.V_{\mathrm{NC}}, V_{\mathrm{NC}}, V_{\mathrm{NC}}, V_{\mathrm{CC}}+V_{\mathrm{NC}}, V_{\mathrm{NC}}, V_{\mathrm{NC}}\right)$. The charged current potential is given by $V_{\mathrm{CC}}=2 E \sqrt{2} G_{F} n_{e}$, where $G_{F}$ is the Fermi constant and $n_{e}$ is the electron number density. The neutral current potential, $V_{\mathrm{NC}}$, is a common term to all the diagonal entries, and therefore, it can be removed from the effective Hamiltonian, which will read as follows:

$$
\mathbb{A}=\operatorname{diag}\left(V_{\mathrm{CC}}, 0,0, V_{\mathrm{CC}}, 0,0\right) .
$$

This Hamiltonian will lead to a different oscillation behavior compared to the standard case, as soon as any $\epsilon_{i}$ or any nonstandard mixing angle is different from zero. As an example, we show in Fig. 1 (top panels) the oscillation probabilities for the two channels relevant for DUNE, $\nu_{\mu} \rightarrow \nu_{e}$ and $\nu_{\mu} \rightarrow \nu_{\mu}$. The standard oscillation parameters in these plots are fixed to the ones in Table I, taken from Ref. [1]. In the left panel we show the disappearance probability $P_{\mu \mu}$ as a function of the neutrino energy, turning on one new mixing angle at a time-which is always set to $\sin ^{2} \theta_{\text {new }}=0.2$. The new angle $\theta_{16}$ has no visible effect on the disappearance probability, while $\theta_{26}$ has a visible effect close to the oscillation minima. In the right panel of Fig. 1, we show the appearance probability $P_{\mu e}$. Here both angles have a visible impact on the oscillation probability. This is expected from the fact that the new angles $\theta_{16}$ and $\theta_{26}$ take the role of the standard angles $\theta_{13}$ and $\theta_{23}$, respectively. On the other hand, the

\footnotetext{
${ }^{1}$ From this expression it is clear why the convention chosen in Eq. (9) is useful. Setting $\epsilon_{i}^{2}$ and the nonstandard angles to zero, $P\left(\nu_{\alpha} \rightarrow \nu_{\beta}\right)$ reduces to the standard expression for three generations, despite the fact that we sum over six states.
} 

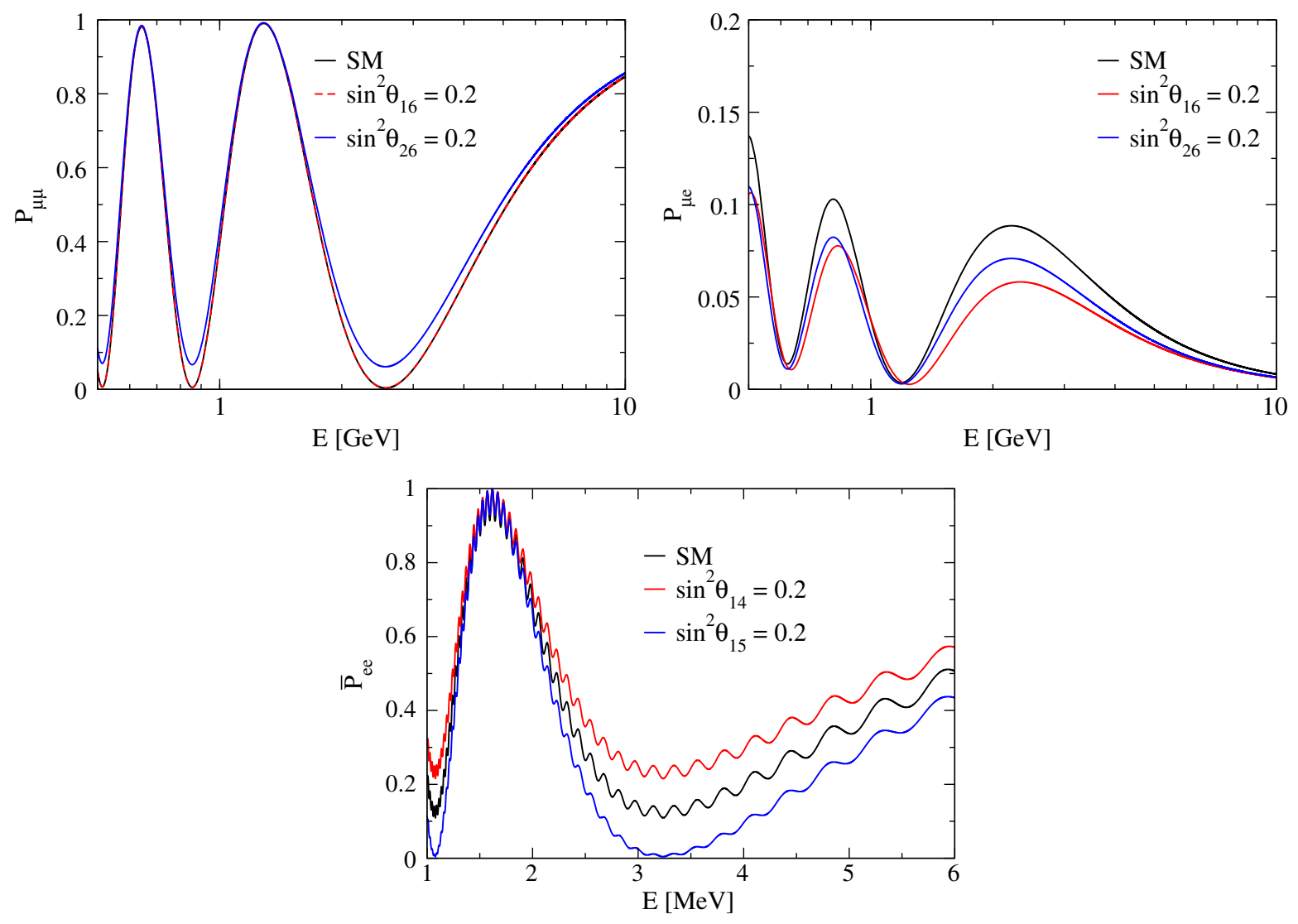

FIG. 1. Disappearance (upper left) and appearance (upper right) probability, $P_{\mu \mu}$ and $P_{\mu e}$, at DUNE as a function of the neutrino energy. Lower panel: Antineutrino survival probability $\bar{P}_{e e}$ in JUNO. In all cases, the black line corresponds to the expected probability in the SM with only three active neutrinos, while the red and blue curves are obtained by setting a new mixing angle in the quasi-Dirac scenario to the value indicated in the legend. All the other new mixing angles are set to zero.

lower panel of Fig. 1 shows the effect of the new mixing angles $\theta_{14}$ and $\theta_{15}$ on the survival probability of electron antineutrinos at JUNO. In this case, the two mixing angles have opposite effects. Note that the survival probability shown here does not include the experimental energy resolution, which is included in our simulation of JUNO in Sec. III.

Figure 1 is meant for illustration purposes only: As we shall see later in Sec. IV, standard and nonstandard angles are highly correlated in the quasi-Dirac neutrino scenario, and one can obtain perfect degeneracies among certain

TABLE I. Standard neutrino oscillation parameters used in the analysis, taken from Ref. [1].

\begin{tabular}{lc}
\hline \hline Parameter & Value \\
\hline$\Delta m_{21}^{2}$ & $7.5 \times 10^{-5} \mathrm{eV}^{2}$ \\
$\Delta m_{31}^{2}$ & $2.50 \times 10^{-3} \mathrm{eV}^{2}$ \\
$\sin ^{2} \theta_{12}$ & 0.32 \\
$\sin ^{2} \theta_{23}$ & 0.547 \\
$\sin ^{2} \theta_{13}$ & 0.0216 \\
$\delta$ & $1.5 \pi$ \\
\hline \hline
\end{tabular}

parameters. That is, even very different combinations of angles can lead to similar oscillation probabilities, which makes the establishment of limits on quasi-Dirac angles particularly difficult experimentally. For this reason, Ref. [5] introduced a particular set of variables, $X_{i}$, which are parametrization-independent combinations of entries in the neutrino mixing matrix $\mathbf{U}$. Not considering transitions to $\nu_{\tau}$, due to the scarcity of $\nu_{\tau}$ appearance data, one can show that only seven independent combinations of neutrino mixing angles enter the oscillation probabilities. The corresponding $X_{i}$ are defined as

$X_{1}=\left|\mathbf{U}_{e 3}\right|^{2}+\left|\mathbf{U}_{e 6}\right|^{2}, \quad X_{2}=\left|\mathbf{U}_{e 2}\right|^{2}+\left|\mathbf{U}_{e 5}\right|^{2}$,

$X_{3}=\left|\mathbf{U}_{\mu 3}\right|^{2}+\left|\mathbf{U}_{\mu 6}\right|^{2}, \quad X_{4}=\left|\mathbf{U}_{\mu 2}\right|^{2}+\left|\mathbf{U}_{\mu 5}\right|^{2}$,

$X_{5}=\left|\mathbf{U}_{e 3} \mathbf{U}_{\mu 3}^{*}+\mathbf{U}_{e 6} \mathbf{U}_{\mu 6}^{*}\right|^{2}, \quad X_{6}=\left|\mathbf{U}_{e 2} \mathbf{U}_{\mu 2}^{*}+\mathbf{U}_{e 5} \mathbf{U}_{\mu 5}^{*}\right|^{2}$,

$X_{7}=\left(\mathbf{U}_{e 3} \mathbf{U}_{\mu 3}^{*}+\mathbf{U}_{e 6} \mathbf{U}_{\mu 6}^{*}\right)\left(\mathbf{U}_{e 2} \mathbf{U}_{\mu 2}^{*}+\mathbf{U}_{e 5} \mathbf{U}_{\mu 5}^{*}\right)$,

where $\mathbf{U}$ is the full mixing matrix defined in Eq. (9). Note that $\left|X_{7}\right|^{2}=X_{5} X_{6}$; i.e., only the phase in $X_{7}$ is a free parameter. The oscillation probabilities in vacuum can be written in terms of the $X_{i}$ as [5] 


$$
\begin{gathered}
P\left(\nu_{e} \rightarrow \nu_{e}\right)=1+\left(1-X_{1}-X_{2}\right) X_{2} \mathcal{A}_{21}+\left(1-X_{1}-X_{2}\right) X_{1} \mathcal{A}_{31}+X_{1} X_{2} \mathcal{A}_{32}, \\
P\left(\nu_{\mu} \rightarrow \nu_{\mu}\right)=1+\left(1-X_{3}-X_{4}\right) X_{4} \mathcal{A}_{21}+\left(1-X_{3}-X_{4}\right) X_{3} \mathcal{A}_{31}+X_{3} X_{4} \mathcal{A}_{32}, \\
P\left(\nu_{e} \rightarrow \nu_{\mu}\right)=-\left(X_{6}+\operatorname{Re} X_{7}\right) \mathcal{A}_{21}-\left(X_{5}+\operatorname{Re} X_{7}\right) \mathcal{A}_{31}+\operatorname{Re} X_{7} \mathcal{A}_{32}+\operatorname{Im} X_{7}\left(\mathcal{B}_{21}-\mathcal{B}_{31}+\mathcal{B}_{32}\right),
\end{gathered}
$$

where $\mathcal{A}_{i j} \equiv-4 \sin ^{2}\left[\left(m_{i}^{2}-m_{j}^{2}\right) L /(4 E)\right]$ and $\mathcal{B}_{i j} \equiv 2 \sin \left[\left(m_{i}^{2}-m_{j}^{2}\right) L /(2 E)\right]$.

The usefulness of defining these $X_{i}$ lies in the fact that, for a three-generation Dirac scenario, there are only four independent parameters entering these seven quantities: the three standard mixing angles and the phase $\delta_{13}$. Thus, in the Dirac limit, one can find three relations among the seven $X_{i}$ :

$$
\begin{aligned}
X_{5} & =X_{1} X_{3}, \quad X_{6}=X_{2} X_{4}, \\
\operatorname{Re}\left(X_{7}\right) & =\frac{1}{2}\left(1-X_{1}-X_{2}-X_{3}-X_{4}+X_{1} X_{4}+X_{2} X_{3}\right) .
\end{aligned}
$$

Equation (18) allows us to formulate quantitative tests of "quasi-Diracness." We will come back to this in Sec. IV. Here we note that, although seven $X_{i}$ are defined here, DUNE will not be sensitive to $X_{1}$ and $X_{2}$ since they depend on the solar parameters. However, JUNO (and Daya Bay, which we will include as a prior in our analysis) will provide stringent constraints on $X_{1}$ and $X_{2}$; see Sec. IV. On the contrary, DUNE will be able to put severe restrictions on $X_{3}$ and $X_{5}$ and some improvements on the remaining parameters $X_{4}, X_{6}$ and $X_{7}$, as we will show below.

Beyond the new mixing angles, we show DUNE's sensitivity to the new mass splittings $\epsilon_{i}$ in Fig. 2. These results have been obtained by varying only one of the new

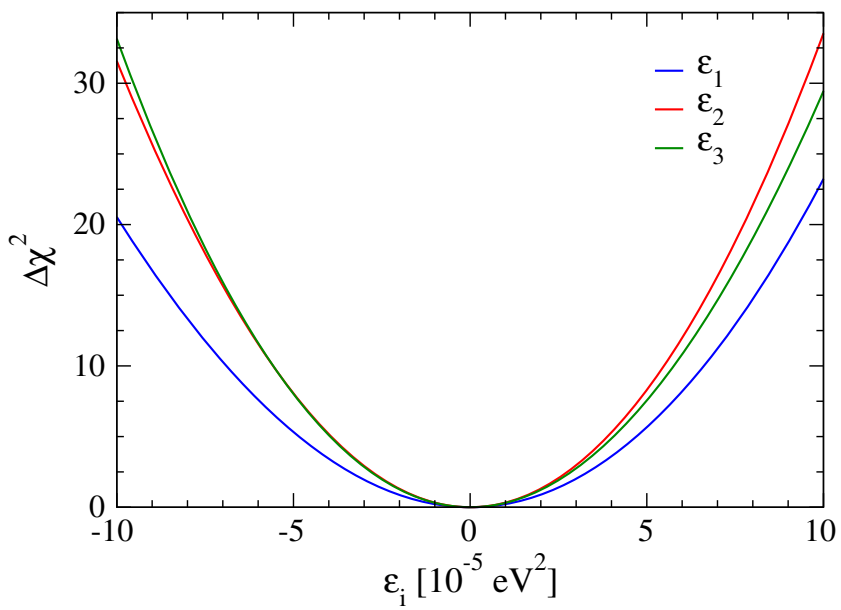

FIG. 2. $\chi^{2}$ profiles for the new mass splittings $\epsilon_{i}$. New angles are fixed to zero. mass splittings at a time and fixing the new angles to zero. In comparison with previous results derived in Ref. [5], one can see that DUNE will not be competitive with other current experiments, which give bounds on $\epsilon_{1}$ and $\epsilon_{2}$ several orders of magnitude stronger than the ones shown in Fig. 2. The only comparable bound is the one for $\epsilon_{3}$. Note, however, that in Ref. [5] the authors marginalized over some of the oscillation parameters while we kept all of them fixed. Marginalizing over additional parameters would result in weaker bounds, also for $\epsilon_{3}$. Therefore, given the poor sensitivity of DUNE to the new splittings $\epsilon_{i}$, we will set them to very small values in our analysis. The sensitivity of JUNO to the mass splittings $\epsilon_{i}$ has been discussed in Ref. [5]. We can infer from Table 1 in [5] that JUNO will not be able to improve the current bounds on any of the new mass splittings.

\section{SIMULATION OF EXPERIMENTS}

The Deep Underground Neutrino Experiment [62-64] is one of the next-generation long-baseline accelerator experiments. It will consist of two detectors exposed to a megawattscale neutrino beam produced at Fermilab. This beam will consist of (nearly) only muon neutrinos. The near detector will be placed approximately 600 meters away from the source of the beam. The second (far) detector, divided into four modules, each using 10 kton of argon as detection material, will be installed 1300 kilometres away deep underground at the Sanford Underground Research Facility in South Dakota.

To simulate the neutrino signal in DUNE, we use the GLoBES package [81,82] with the configuration file provided by the DUNE Collaboration [83]. We assume DUNE to run 3.5 years in the neutrino mode and another 3.5 years in the antineutrino mode. Considering an $80 \mathrm{GeV}$ beam with $1.07 \mathrm{MW}$ beam power, this corresponds to an exposure of 300 kton-MW-years. In this configuration, DUNE will be using $1.47 \times 10^{21}$ protons on target $(\mathrm{POT})$ per year. Our analysis includes disappearance and appearance channels, simulating both signals and backgrounds. The simulated backgrounds include contamination of antineutrinos (neutrinos) in the neutrino (antineutrino) mode and also misinterpretation of flavors.

To include quasi-Dirac neutrino oscillations in our simulation of DUNE, we use the GLoBES extension 
snu. C $[84,85]$. This extension was originally made to include nonstandard neutrino interactions and sterile neutrinos in GLoBES simulations. For this analysis, we have modified the definition of the neutrino oscillation probability function inside snu. $\mathrm{C}$ by adding the additional rotation matrix of Eq. (9) and the matter potential of Eq. (13).

For the statistical analysis, we create a fake DUNE data sample using the standard oscillation parameters from Table I. Next, we try to reconstruct the simulated data varying the mixing angles $\theta_{13}, \theta_{23}, \theta_{16}$ and $\theta_{26}$ (most relevant for DUNE) and the two $C P$-violating phases $\delta_{13}$ and $\delta_{16}$. The remaining new mixing angles are fixed to zero, and the new mass splittings $\epsilon_{i}^{2}$ are fixed to very small values. Note as well that, since DUNE has no sensitivity to the solar parameters, these are fixed at their best-fit values in Table I. On the other hand, given that we are mostly interested in correlations between the standard and new mixing angles, we have also kept $\Delta m_{31}^{2}$ fixed to its best-fit value. Currently, there is a preference for normal mass ordering slightly above $3 \sigma[1,86,87]$, so we will not consider negative values of $\Delta m_{31}^{2}$ here. We use GLoBES to calculate the event numbers for a given set of oscillation parameters $p$, and then we calculate the $\chi^{2}$ value for this set using the following expression:

$$
\chi_{\mathrm{DUNE}}^{2}(p)=\min _{\vec{\alpha}} \sum_{\text {channels }} 2 \sum_{n}\left[N_{n}(p, \vec{\alpha})-N_{n}^{\mathrm{dat}}+N_{n}^{\mathrm{dat}} \log \left(\frac{N_{n}^{\mathrm{dat}}}{N_{n}(p, \vec{\alpha})}\right)\right]+\sum_{i}\left(\frac{\alpha_{i}}{\sigma_{i}}\right)^{2} .
$$

Here, $N_{n}^{\text {dat }}$ corresponds to the simulated event number in the $n$th bin for the oscillation parameters in Table I, and $N_{n}(p, \vec{\alpha})$ is the event number predicted in the $n$th bin associated to the oscillation parameters $p$ and to the nuisance parameters $\alpha_{i}$, with standard deviations given by $\sigma_{i}$. All the nuisance parameters are associated to normalization uncertainties of signal or background events and introduce modifications of the type $N_{n} \rightarrow N_{n}\left(1+\alpha_{i}\right)$. The last term in Eq. (19) penalizes the deviation of the latter parameters from their expectation values, $\alpha_{i}=0$. Finally, the $\chi^{2}$ sums over disappearance and appearance channels in both neutrino and antineutrino modes. The simulation and sensitivity analysis of the JUNO reactor experiment are performed following the procedure described in [5]. The corresponding $\chi^{2}$ function, $\chi_{\text {JUNO }}^{2}$, is obtained by allowing the variation of only five parameters which are relevant for JUNO, namely, $\theta_{12}, \theta_{13}, \theta_{14}, \theta_{15}$ and $\theta_{16}$. The solar mass splitting $\Delta m_{21}^{2}$ is fixed to the best-fit value. To get the global future sensitivity to the quasi-Dirac scenario, in our analysis we combine the individual sensitivities obtained for DUNE and JUNO. Besides the two $\chi^{2}$ functions discussed above, $\chi_{\text {DUNE }}^{2}$ and $\chi_{\text {JUNO }}^{2}$, we introduce a penalty function associated to some of the mixing angles under study. As it was shown in [5], the current reactor experiments cannot univocally measure the reactor angle $\theta_{13}$ in the presence of quasi-Dirac neutrinos. However, it is still possible to simultaneously constrain several of these angles. If not, Daya Bay would have observed a different signal. This penalty can be obtained from Eq. (15) by imposing $\left(1-X_{1}-X_{2}\right) X_{1}+X_{1} X_{2}=\sin ^{2} \theta_{\mathrm{DB}}$ where $\sin ^{2} \theta_{\mathrm{DB}} \approx 0.022$ is the value currently measured by the Daya Bay reactor experiment [88]. Hence, our global $\chi^{2}$ function can be written as

$$
\chi^{2}(p)=\chi_{\mathrm{DUNE}}^{2}(p)+\chi_{\mathrm{JUNO}}^{2}(p)+f_{\mathrm{DB}}(p) .
$$

The penalty function in terms of the relevant mixing angles is given by

$$
f_{\mathrm{DB}}(p)=\left[\frac{\left(\left(c_{14} c_{15} c_{16} s_{13}\right)^{2}+s_{16}^{2}-1\right)\left(\left(c_{14} c_{15} c_{16} s_{13}\right)^{2}+s_{16}^{2}\right)-\sin ^{2} \theta_{\mathrm{DB}}}{\sigma_{\mathrm{DB}} \sin ^{2} \theta_{\mathrm{DB}}}\right]^{2},
$$

where $c_{i j}=\cos \theta_{i j}, s_{i j}=\sin \theta_{i j}$ and $\sigma_{\mathrm{DB}}$ is the expected uncertainty in the final measurement of the reactor mixing angle by Daya Bay, set to 3\%. This is a generalization of the standard reactor prior used in several studies on neutrino oscillations.

\section{RESULTS AND DISCUSSION}

In this section, we present the results of the statistical analysis performed in this work. Before discussing the results of the combined analysis of DUNE and JUNO, we discuss the results of the two experiments separately. Note, however, that we always add the penalty term in Eq. (21) to the $\chi^{2}$ function obtained from the sensitivity analysis of each experiment. In Fig. 3 we show the two-dimensional allowed regions obtained by scanning over the parameters $\theta_{13}, \theta_{23}, \theta_{16}, \theta_{26}, \delta_{13}$ and $\delta_{16}$ in DUNE. The parameters not shown are marginalized over in each panel. The colored regions correspond to the 1 (cyan), 2 (blue), 3 (red) $\sigma$ confidence levels for 2 degrees of freedom. In the upper 

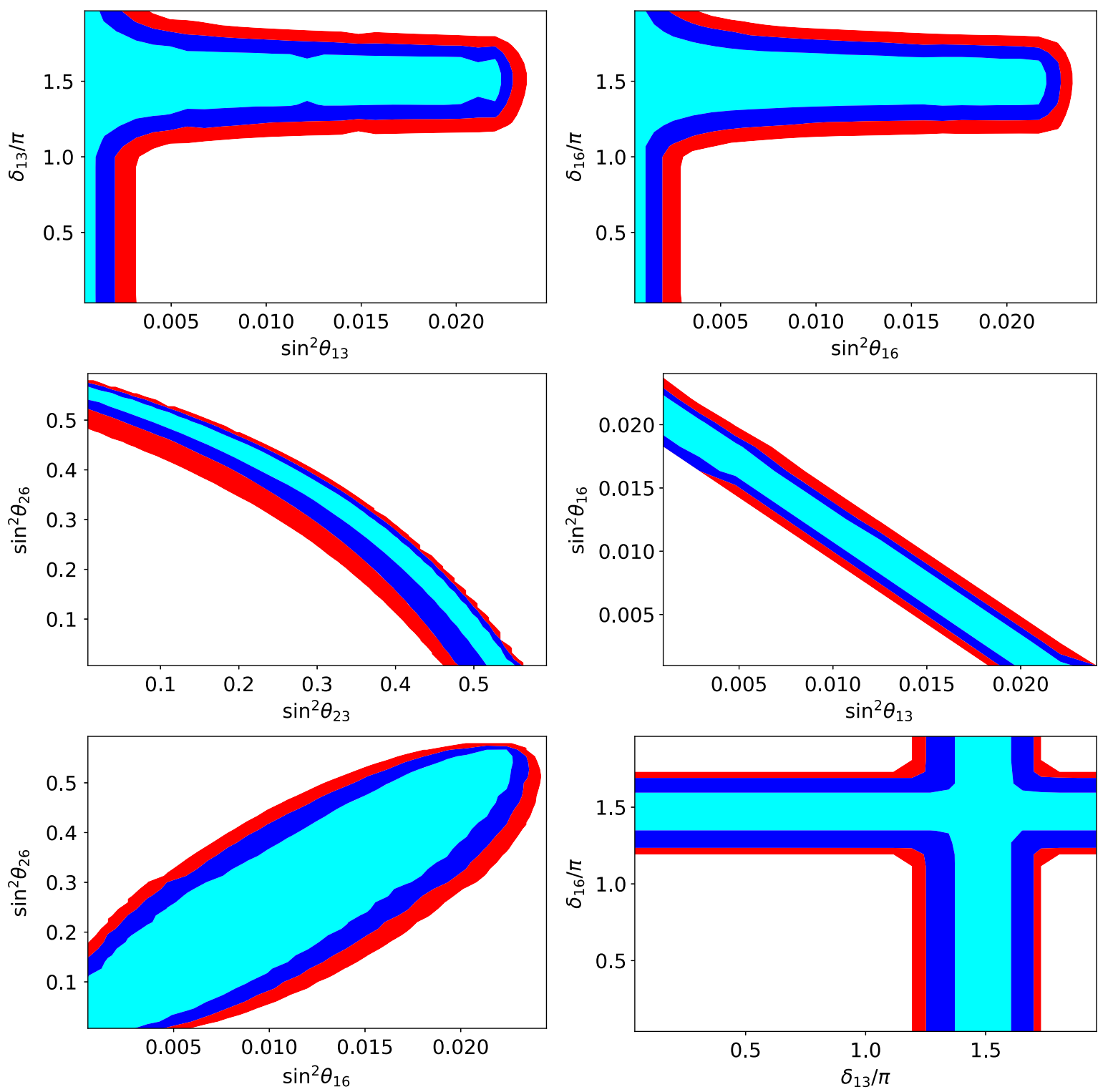

FIG. 3. DUNE sensitivity to the oscillation parameters under study. The colored regions shown correspond to 1 (cyan), 2 (blue), 3 (red) $\sigma$ confidence levels for 2 degrees of freedom. In each two-dimensional plot we have marginalized over the other parameters which are not displayed.

panels, we see that the two reactor angles, $\theta_{13}$ and $\theta_{16}$, and their corresponding phases, $\delta_{13}$ and $\delta_{16}$, behave in a very similar way. In principle, small values of the phases are allowed, although these require very small values for the associated mixing angles. From the right panel of the second row, however, we see that both angles cannot be small at the same time: if $\theta_{13}$ is small, $\theta_{16}$ has to be large, and vice versa. In the former case, $\delta_{13}$ can take any value in the interval $[0,2 \pi]$, while $\delta_{16}$ is rather restricted around its maximal value $1.5 \pi$. This is an interesting point because the fake data were created with $\delta_{16}=0$. The reason behind this is that the new angles and phases are correlated to the standard angles, e.g., $\theta_{13}$ and $\theta_{16}$ [see the definitions in Eq. (14)]; hence they are interchangeable. Note as well that all the sensitivity to the reactor angle is lost since DUNE can only reproduce the prior [5] that we introduced as an input for our analysis, as explained in Sec. III. The interchangeability of the mixing parameters can also be seen from the left panel of the second row in Fig. 3. There, we see that $\theta_{23}$ and $\theta_{26}$ are also fully correlated: having a large $\theta_{23}$ and a small $\theta_{26}$ is equivalent to having a small $\theta_{23}$ and a large $\theta_{26}$. The same applies to the $C P$-violating phases, as can be seen in the right panel of the last row. The left panel of the last row shows that there are correlations also between the atmospheric and reactor angles, which are not present in the standard case of three-neutrino oscillations anymore, given the very good level of precision achieved in the determination of the mixing angles. 


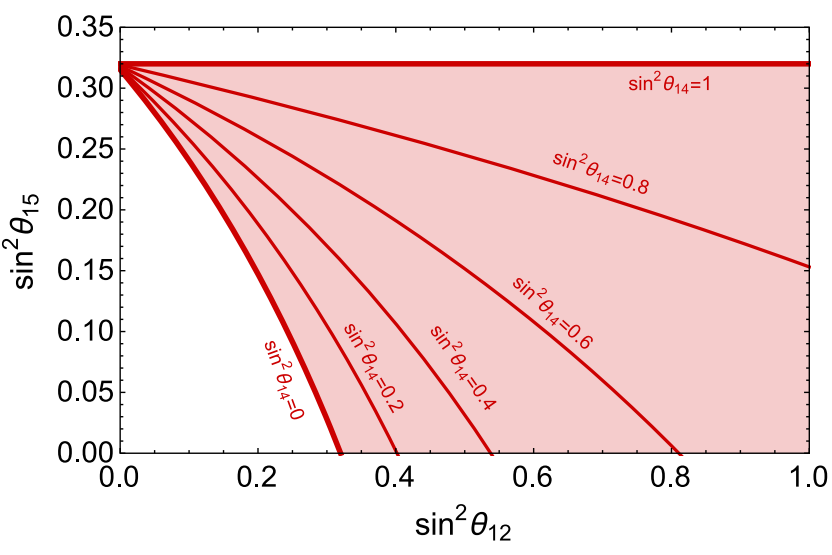

FIG. 4. Sensitivity region in the $\left(\sin ^{2} \theta_{12}, \sin ^{2} \theta_{15}\right)$ plane for the JUNO experiment. The shaded region corresponds to the $1 \sigma$ allowed region once marginalized over $\sin ^{2} \theta_{14}$. See the text for more details.

Since $\theta_{16}$ and $\theta_{13}$, as well as $\theta_{26}$ and $\theta_{23}$, are equivalent, a similar result is obtained in the two-dimensional plane $\left(\theta_{13}, \theta_{23}\right)$.

In Fig. 4 we show the result of our simulation of JUNO. In this case, we find that the new angles $\theta_{14}$ and $\theta_{15}$ are highly correlated with the standard solar angle $\theta_{12}$. In particular, one sees that, for $\sin ^{2} \theta_{14}=0$, all values of $\theta_{12}$ and $\theta_{15}$ lying along the correspondingly labeled line are possible, showing a similar correlation as in the case of $\theta_{13}$ and $\theta_{16}$ or $\theta_{23}$ and $\theta_{26}$. For different values of $\sin ^{2} \theta_{14}$ the correlating line is shifted as indicated in the figure. If we now marginalize over all possible values for $\theta_{14}$, we find that a large region of parameter space is still allowed. It is however important to notice that a point in the $\left(\sin ^{2} \theta_{12}\right.$, $\sin ^{2} \theta_{15}$ ) plane always corresponds to one specific value of $\sin ^{2} \theta_{14}$.

Now let us discuss the results of the combined analysis of DUNE and JUNO in terms of the variables $X_{i}$ introduced in Eq. (14). Compared to Ref. [5], where most of the parameter space was allowed by current neutrino oscillation data, here we find that DUNE and JUNO will be able to strongly constrain some of the $X_{i}$ parameters. This is illustrated in Fig. 5, where we show the $\Delta \chi^{2}$ profiles for the $X_{i}$ variables. In the left panel, we see how precise DUNE and JUNO could measure some of these quantities. Notably, $X_{1}, X_{2}$ and $X_{3}$ can be measured with a precision below $\%$. The sensitivity to $X_{4}, X_{5}, X_{6}$ and $X_{7}$ will also be improved with respect to the current results obtained in [5], although not as dramatically as for the previous three parameters. Note, however, that DUNE will not be able to set a lower limit on $X_{4}, X_{6}$ and $X_{7}$, which are allowed to be zero in our combined fits. In the case of $X_{4}$, this can be traced to the fact that DUNE does not have the resolution to demonstrate that there are three independent oscillation frequencies contributing to $P\left(\nu_{\mu} \rightarrow \nu_{\mu}\right)$ [see Eq. (16)]. An upper limit on $X_{4}$ can instead be obtained from the unitarity relation $X_{3}+X_{4}<1$. Similar comments apply to $X_{6}$ and $X_{7}$.

In the right panel of Fig. 5 , we construct a quantity to directly test the Diracness of neutrino oscillations. This quantity is obtained by assuming that neutrinos are Dirac particles in Eq. (14); see Ref. [5] for more details. In this case one can derive

$$
1-\frac{X_{5}}{X_{1} X_{3}}=0
$$

Any deviation from zero in this expression would be an indication for quasi-Dirac neutrinos. Since we created our fake data assuming neutrinos to be Dirac particles, our bestfit point is automatically located at zero. However, DUNE could restrict the allowed deviation considerably, as shown in the plot.

Finally, to further investigate the discrimination power of the experiments to the quasi-Dirac scenario, we have created another fake data set using a quasi-Dirac point as an input. For the particular point we have chosen, we expect
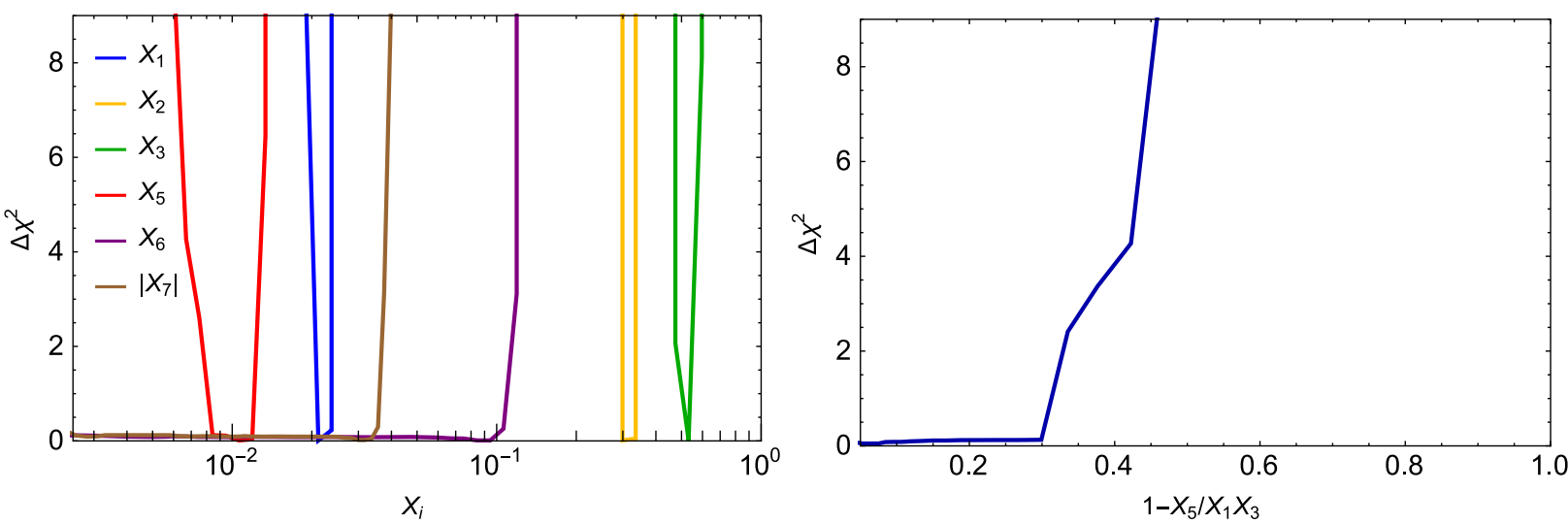

FIG. 5. Left panel: $\chi^{2}$ profiles for the $X_{i}$ variables. Right panel: Diracness test for a Dirac input point. 

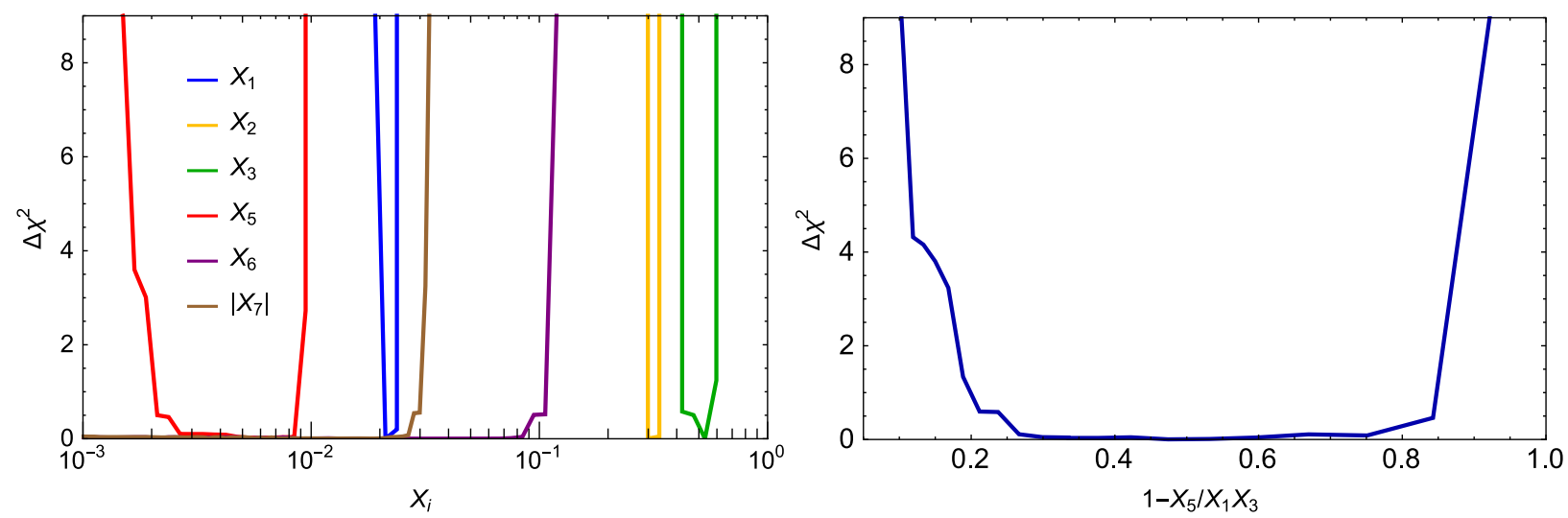

FIG. 6. Left panel: $\chi^{2}$ profiles for the $X_{i}$ variables. Right panel: Diracness test for a quasi-Dirac input point.

$$
1-\frac{X_{5}}{X_{1} X_{3}}=0.5
$$

Our choice falls inside the $1 \sigma$ contours of Fig. 3, and it corresponds to $\sin ^{2} \theta_{23}=0.30, \sin ^{2} \theta_{26}=0.37$, $\sin ^{2} \theta_{13}=0.0108, \sin ^{2} \theta_{16}=0.0108$. The $C P$-violating phases are assumed as in the first analysis. The result for this simulation is shown in Fig. 6. In the left panel one can see how most of the $X_{i}$ are mostly unaffected by the selected input point, while there is a visible difference in the profiles corresponding to $X_{3}$ and $X_{5}$. Nevertheless, the most visible effect appears in the right panel of the figure. There, we see that the Dirac point—with $1-\frac{X_{5}}{X_{1} X_{3}}=0$ - could be completely excluded in this scenario. This is an important result because it means that DUNE and JUNO would be able to distinguish standard three-neutrino oscillations from quasi-Dirac oscillations. Note, however, that this statement is true for our benchmark point. If the true value lies very close to Diracness, it would be more difficult to discriminate between the two scenarios.

\section{CONCLUSIONS}

We have studied the sensitivity of the DUNE and JUNO experiments to quasi-Dirac neutrino oscillations. We have found that, within this scenario, the determination of neutrino mixing angles becomes much more complicated, if not impossible, even for next-generation experiments such as DUNE or JUNO. The main reason is that the new angles and phases are strongly correlated to the corresponding ones in the active sector, leading to very relevant degeneracies.

As a further comment, let us mention that many of the degeneracies observed here could be broken by including a $\nu_{\tau}$ appearance channel in the DUNE analysis. This possibility has been recently discussed in Refs. [89,90]. If neutrinos are quasi-Dirac particles, $\sum_{\beta} P_{\alpha \beta}<1$, with $\beta=\{e, \mu, \tau\}$. Hence, a more precise observation of the unitarity of neutrino oscillations including the $\nu_{\tau}$ channel would be extremely helpful to test the quasi-Dirac neutrino hypothesis, as well as other nonunitary neutrino scenarios [91,92].

Despite the degeneracies affecting the angles, we have seen that we can define new observables which clearly allow us to distinguish the standard oscillation case from the quasi-Dirac neutrino scenario. While most of the parameter space for these observables is still allowed at present, we have shown that DUNE and JUNO can considerably improve the current bounds on these quantities. We have also seen that, if quasi-Dirac neutrino oscillations are real, the new generation of experiments will have the potential to discover quasi-Dirac neutrinos, which would be a big breakthrough in particle physics.

\section{ACKNOWLEDGMENTS}

We would like to thank Stefano Gariazzo and Renato Fonseca for useful discussions. This work is supported by the Spanish Grants No. FPA2017-90566-REDC (Red Consolider MultiDark), No. FPA2017-85216-P and No. SEV-2014-0398 (MINECO/AEI/FEDER, UE), as well as PROMETEO/2018/165 (Generalitat Valenciana). V. D. R. acknowledges financial support by the "Juan de la Cierva Incorporación" program (IJCI-2016-27736) funded by the Spanish MINECO. C. A. T. is also supported by the FPI (Formación de Personal Investigador) Contract No. BES-2015-073593. M. T. acknowledges financial support from MINECO through the Ramón y Cajal Contract No. RYC-2013-12438. 
[1] P. F. de Salas, D. V. Forero, C. A. Ternes, M. Tortola, and J. W. F. Valle, Status of neutrino oscillations 2018: $3 \sigma$ hint for normal mass ordering and improved $C P$ sensitivity, Phys. Lett. B 782, 633 (2018).

[2] S. Gariazzo, C. Giunti, M. Laveder, and Y. F. Li, Updated global $3+1$ analysis of short-baseline neutrino oscillations, J. High Energy Phys. 06 (2017) 135.

[3] M. Dentler, A. Hernandez-Cabezudo, J. Kopp, P. A. N. Machado, M. Maltoni, I. Martinez-Soler, and T. Schwetz, Updated global analysis of neutrino oscillations in the presence of eV-scale sterile neutrinos, J. High Energy Phys. 08 (2018) 010.

[4] I. Esteban, M. C. Gonzalez-Garcia, M. Maltoni, I. MartinezSoler, and J. Salvado, Updated constraints on non-standard interactions from global analysis of oscillation data, J. High Energy Phys. 08 (2018) 180.

[5] G. Anamiati, R. M. Fonseca, and M. Hirsch, Quasi Dirac neutrino oscillations, Phys. Rev. D 97, 095008 (2018).

[6] F. T. Avignone III, S. R. Elliott, and J. Engel, Double beta decay, Majorana neutrinos, and neutrino mass, Rev. Mod. Phys. 80, 481 (2008).

[7] F. F. Deppisch, M. Hirsch, and H. Pas, Neutrinoless double beta decay and physics beyond the Standard Model, J. Phys. G 39, 124007 (2012).

[8] J. W. F. Valle, Neutrinoless double beta decay with quasi Dirac neutrinos, Phys. Rev. D 27, 1672 (1983).

[9] L. Wolfenstein, Different varieties of massive Dirac neutrinos, Nucl. Phys. B186 (1981) 147.

[10] S. T. Petcov, On pseudo-Dirac neutrinos, neutrino oscillations and neutrinoless double beta decay, Phys. Lett. 110B, 245 (1982).

[11] M. Doi, M. Kenmoku, T. Kotani, H. Nishiura, and E. Takasugi, Pseudo-Dirac neutrino, Prog. Theor. Phys. 70, 1331 (1983).

[12] S. M. Bilenky and B. Pontecorvo, Neutrino oscillations with large oscillation length in spite of large (Majorana) neutrino masses?, Yad. Fiz. 38, 415 (1983) [Sov. J. Nucl. Phys. 38, 248 (1983)].

[13] S. M. Bilenky and S. T. Petcov, Massive neutrinos and neutrino oscillations, Rev. Mod. Phys. 59, 671 (1987); Erratum, Rev. Mod. Phys. 60, 575(E) (1988).

[14] C. Giunti, C. W. Kim, and U. W. Lee, Oscillations of pseudo-Dirac neutrinos and the solar neutrino problem, Phys. Rev. D 46, 3034 (1992).

[15] G. Dutta and A. S. Joshipura, Pseudo-Dirac neutrinos in seesaw model, Phys. Rev. D 51, 3838 (1995).

[16] A. S. Joshipura and S. D. Rindani, Phenomenology of pseudo-Dirac neutrinos, Phys. Lett. B 494, 114 (2000).

[17] Y. Nir, Pseudo-Dirac solar neutrinos, J. High Energy Phys. 06 (2000) 039.

[18] B. Brahmachari and S. Choubey, Viability of bimaximal solution of the Zee mass matrix, Phys. Lett. B 531, 99 (2002).

[19] P. H. Frampton, M. C. Oh, and T. Yoshikawa, Zee model confronts SNO data, Phys. Rev. D 65, 073014 (2002).

[20] X.-G. He, Is the Zee model neutrino mass matrix ruled out?, Eur. Phys. J. C 34, 371 (2004).

[21] A. Geiser, Pseudo-Dirac neutrinos as a potential complete solution to the neutrino oscillation puzzle, Phys. Lett. B 444, 358 (1998).
[22] W. Krolikowski, Option of three pseudo-Dirac neutrinos, Acta Phys. Pol. B 31, 663 (2000).

[23] K. R. S. Balaji, A. Kalliomaki, and J. Maalampi, Revisiting pseudo-Dirac neutrinos, Phys. Lett. B 524, 153 (2002).

[24] E. Ma and P. Roy, Model of four light neutrinos in the light of all present data, Phys. Rev. D 52, R4780 (1995).

[25] S. Goswami and A.S. Joshipura, Neutrino anomalies and quasi Dirac neutrinos, Phys. Rev. D 65, 073025 (2002).

[26] M. Cirelli, G. Marandella, A. Strumia, and F. Vissani, Probing oscillations into sterile neutrinos with cosmology, astrophysics and experiments, Nucl. Phys. B708, 215 (2005).

[27] A. de Gouvea, W.-C. Huang, and J. Jenkins, Pseudo-Dirac neutrinos in the new Standard Model, Phys. Rev. D 80, 073007 (2009).

[28] J. F. Beacom, N. F. Bell, D. Hooper, J. G. Learned, S. Pakvasa, and T.J. Weiler, Pseudo-Dirac Neutrinos: A Challenge for Neutrino Telescopes, Phys. Rev. Lett. 92, 011101 (2004).

[29] A. Esmaili, Pseudo-Dirac neutrino scenario: Cosmic neutrinos at neutrino telescopes, Phys. Rev. D 81, 013006 (2010).

[30] A. Esmaili and Y. Farzan, Implications of the pseudo-Dirac scenario for ultra high energy neutrinos from GRBs, J. Cosmol. Astropart. Phys. 12 (2012) 014.

[31] A. S. Joshipura, S. Mohanty, and S. Pakvasa, Pseudo-Dirac neutrinos via a mirror world and depletion of ultrahigh energy neutrinos, Phys. Rev. D 89, 033003 (2014).

[32] G. J. Stephenson, Jr., J. T. Goldman, B. H. J. McKellar, and M. Garbutt, Large mixing from small: Pseudo-Dirac neutrinos and the singular seesaw, Int. J. Mod. Phys. A 20, 6373 (2005).

[33] K. L. McDonald and B. H. J. McKellar, The type-II singular see-saw mechanism, Int. J. Mod. Phys. A 22, 2211 (2007).

[34] Y. H. Ahn, S. K. Kang, and C. S. Kim, A model for pseudoDirac neutrinos: Leptogenesis and ultra-high energy neutrinos, J. High Energy Phys. 10 (2016) 092.

[35] D. Chang and O.C.W. Kong, Pseudo-Dirac neutrinos, Phys. Lett. B 477, 416 (2000).

[36] L. A. Sanchez, W. A. Ponce, and R. Martinez, $S U(3)_{c} \otimes$ $S U(3)_{L} \otimes U(1)_{X}$ as an $E_{6}$ subgroup, Phys. Rev. D 64, 075013 (2001).

[37] R. M. Fonseca and M. Hirsch, Lepton number violation in 331 models, Phys. Rev. D 94, 115003 (2016).

[38] S. Abel, A. Dedes, and K. Tamvakis, Naturally small Dirac neutrino masses in supergravity, Phys. Rev. D 71, 033003 (2005).

[39] C. Athanassopoulos et al. (LSND Collaboration), Evidence for $\bar{\nu}_{\mu} \rightarrow \bar{\nu}_{e}$ Oscillations from the LSND Experiment at the Los Alamos Meson Physics Facility, Phys. Rev. Lett. 77, 3082 (1996).

[40] A. Aguilar-Arevalo et al. (LSND Collaboration), Evidence for neutrino oscillations from the observation of $\bar{\nu}_{e}$ appearance in a $\bar{\nu}_{\mu}$ beam, Phys. Rev. D 64, 112007 (2001).

[41] J. N. Abdurashitov et al., Measurement of the response of a Ga solar neutrino experiment to neutrinos from an Ar-37 source, Phys. Rev. C 73, 045805 (2006).

[42] M. Laveder, Unbound neutrino roadmaps, Nucl. Phys. B, Proc. Suppl. 168, 344 (2007). 
[43] C. Giunti and M. Laveder, Short-baseline activesterile neutrino oscillations?, Mod. Phys. Lett. A 22, 2499 (2007).

[44] G. Mention, M. Fechner, T. Lasserre, T. A. Mueller, D. Lhuillier, M. Cribier, and A. Letourneau, The reactor antineutrino anomaly, Phys. Rev. D 83, 073006 (2011).

[45] S. Gariazzo, C. Giunti, M. Laveder, and Y. F. Li, Modelindependent $\bar{\nu}_{e}$ short-baseline oscillations from reactor spectral ratios, Phys. Lett. B 782, 13 (2018).

[46] M. Dentler, A. Hernandez-Cabezudo, J. Kopp, M. Maltoni, and T. Schwetz, Sterile neutrinos or flux uncertainties?Status of the reactor anti-neutrino anomaly, J. High Energy Phys. 11 (2017) 099.

[47] P. Adamson et al. (NOvA Collaboration), Search for activesterile neutrino mixing using neutral-current interactions in NOvA, Phys. Rev. D 96, 072006 (2017).

[48] Q. R. Ahmad et al. (SNO Collaboration), Direct Evidence for Neutrino Flavor Transformation from Neutral Current Interactions in the Sudbury Neutrino Observatory, Phys. Rev. Lett. 89, 011301 (2002).

[49] M. G. Aartsen et al. (IceCube Collaboration), Search for sterile neutrino mixing using three years of IceCube DeepCore data, Phys. Rev. D 95, 112002 (2017).

[50] P. Adamson et al. (MINOS and Daya Bay Collaboration), Limits on Active to Sterile Neutrino Oscillations from Disappearance Searches in the MINOS, Daya Bay, and Bugey-3 Experiments, Phys. Rev. Lett. 117, 151801 (2016); Addendum, 117, 209901(A) (2016).

[51] N. Agafonova et al. (OPERA Collaboration), Limits on muon-neutrino to tau-neutrino oscillations induced by a sterile neutrino state obtained by OPERA at the CNGS beam, J. High Energy Phys. 06 (2015) 069.

[52] K. Abe et al. (Super-Kamiokande Collaboration), Limits on sterile neutrino mixing using atmospheric neutrinos in Super-Kamiokande, Phys. Rev. D 91, 052019 (2015).

[53] P. Adamson et al. (MINOS Collaboration), Search for Sterile Neutrinos Mixing with Muon Neutrinos in MINOS, Phys. Rev. Lett. 117, 151803 (2016).

[54] P. Adamson et al. (MINOS+ Collaboration), Search for Sterile Neutrinos in MINOS and MINOS+ Using a TwoDetector Fit, Phys. Rev. Lett. 122, 091803 (2019).

[55] A. Albert et al. (ANTARES Collaboration), Measuring the atmospheric neutrino oscillation parameters and constraining the $3+1$ neutrino model with ten years of ANTARES data, J. High Energy Phys. 06 (2019) 113.

[56] K. Abe et al. (T2K Collaboration), Search for light sterile neutrinos with the T2K far detector Super-Kamiokande at a baseline of 295 km, Phys. Rev. D 99, 071103 (2019).

[57] A. Diaz, C. A. Argüelles, G. H. Collin, J. M. Conrad, and M. H. Shaevitz, Where are we with light sterile neutrinos?, arXiv:1906.00045.

[58] S. Gariazzo, C. Giunti, and C. Ternes (to be published).

[59] C. Giunti and E. M. Zavanin, Appearance-disappearance relation in $3+N_{s}$ short-baseline neutrino oscillations, Mod. Phys. Lett. A 31, 1650003 (2016).

[60] C. Giunti and T. Lasserre, eV-scale sterile neutrinos, arXiv:1901.08330.

[61] S. Böser, C. Buck, C. Giunti, J. Lesgourgues, L. Ludhova, S. Mertens, A. Schukraft, and M. Wurm, Status of light sterile neutrino searches, arXiv:1906.01739.
[62] B. Abi et al. (DUNE Collaboration), The DUNE far detector interim design report volume 1: Physics, technology and strategies, arXiv:1807.10334.

[63] B. Abi et al. (DUNE Collaboration), The DUNE far detector interim design report, volume 2: Single-phase module, arXiv:1807.10327.

[64] B. Abi et al. (DUNE Collaboration), The DUNE far detector interim design report, volume 3: Dual-phase module, arXiv:1807.10340.

[65] F. An et al. (JUNO Collaboration), Neutrino physics with JUNO, J. Phys. G 43, 030401 (2016).

[66] M. Ghosh, S. Goswami, and S. K. Raut, Maximizing the DUNE early physics output with current experiments, Eur. Phys. J. C 76, 114 (2016).

[67] V. De Romeri, E. Fernandez-Martinez, and M. Sorel, Neutrino oscillations at DUNE with improved energy reconstruction, J. High Energy Phys. 09 (2016) 030.

[68] R. Srivastava, C. A. Ternes, M. Tórtola, and J. W. F. Valle, Zooming in on neutrino oscillations with DUNE, Phys. Rev. D 97, 095025 (2018).

[69] P. Coloma, Non-standard interactions in propagation at the deep underground neutrino experiment, J. High Energy Phys. 03 (2016) 016.

[70] A. de Gouvêa and K. J. Kelly, Non-standard neutrino interactions at DUNE, Nucl. Phys. B908, 318 (2016).

[71] M. Blennow, S. Choubey, T. Ohlsson, D. Pramanik, and S. K. Raut, A combined study of source, detector and matter non-standard neutrino interactions at DUNE, J. High Energy Phys. 08 (2016) 090.

[72] P. Coloma, D. V. Forero, and S. J. Parke, DUNE sensitivities to the mixing between sterile and tau neutrinos, J. High Energy Phys. 07 (2018) 079.

[73] S. K. Agarwalla, S. S. Chatterjee, and A. Palazzo, Physics reach of DUNE with a light sterile neutrino, J. High Energy Phys. 09 (2016) 016.

[74] F. J. Escrihuela, D. V. Forero, O. G. Miranda, M. Tórtola, and J. W. F. Valle, Probing CP violation with non-unitary mixing in long-baseline neutrino oscillation experiments: DUNE as a case study, New J. Phys. 19, 093005 (2017).

[75] M. Masud, M. Bishai, and P. Mehta, Extricating new physics scenarios at DUNE with higher energy beams, Sci. Rep. 9, 352 (2019).

[76] G. Barenboim, C. A. Ternes, and M. Tórtola, Neutrinos, DUNE and the world best bound on CPT invariance, Phys. Lett. B 780, 631 (2018).

[77] G. Barenboim, C. A. Ternes, and M. Tórtola, New physics vs new paradigms: Distinguishing CPT violation from NSI, Eur. Phys. J. C 79, 390 (2019).

[78] H. Nunokawa, S. J. Parke, and R. Z. Funchal, Another possible way to determine the neutrino mass hierarchy, Phys. Rev. D 72, 013009 (2005).

[79] S. Parke, What is $\Delta m_{e e}^{2}$ ?, Phys. Rev. D 93, 053008 (2016).

[80] J. Schechter and J. W. F. Valle, Neutrino masses in $\mathrm{SU}(2) \otimes$ U(1) theories, Phys. Rev. D 22, 2227 (1980).

[81] P. Huber, M. Lindner, and W. Winter, Simulation of longbaseline neutrino oscillation experiments with GLoBES (General Long Baseline Experiment Simulator), Comput. Phys. Commun. 167, 195 (2005).

[82] P. Huber, J. Kopp, M. Lindner, M. Rolinec, and W. Winter, New features in the simulation of neutrino oscillation 
experiments with GLoBES 3.0: General long baseline experiment simulator, Comput. Phys. Commun. 177, 432 (2007).

[83] T. Alion et al. (DUNE Collaboration), Experiment simulation configurations used in DUNE CDR, arXiv:1606.09550.

[84] J. Kopp, Efficient numerical diagonalization of Hermitian 3 x 3 matrices, Int. J. Mod. Phys. C 19, 523 (2008).

[85] J. Kopp, M. Lindner, T. Ota, and J. Sato, Non-standard neutrino interactions in reactor and superbeam experiments, Phys. Rev. D 77, 013007 (2008).

[86] S. Gariazzo, M. Archidiacono, P. F. de Salas, O. Mena, C. A. Ternes, and M. Tórtola, Neutrino masses and their ordering: Global data, priors and models, J. Cosmol. Astropart. Phys. 03 (2018) 011.

[87] P. F. de Salas, S. Gariazzo, O. Mena, C. A. Ternes, and M. Tórtola, Neutrino mass ordering from oscillations and beyond: 2018 status and future prospects, Front. Astron. Space Sci. 5, 36 (2018).
[88] D. Adey et al. (Daya Bay Collaboration), Measurement of the Electron Antineutrino Oscillation with 1958 Days of Operation at Daya Bay, Phys. Rev. Lett. 121, 241805 (2018).

[89] A. de Gouvêa, K. J. Kelly, G. V. Stenico, and P. Pasquini, Physics with beam Tau-neutrino appearance at DUNE, Phys. Rev. D 100, 016004 (2019).

[90] A. Ghoshal, A. Giarnetti, and D. Meloni, On the role of the $\nu_{\tau}$ appearance in DUNE in constraining standard neutrino physics and beyond, arXiv:1906.06212.

[91] F. J. Escrihuela, D. V. Forero, O. G. Miranda, M. Tortola, and J. W. F. Valle, On the description of nonunitary neutrino mixing, Phys. Rev. D 92, 053009 (2015); Erratum, 93, 119905 (2016).

[92] M. Blennow, P. Coloma, E. Fernandez-Martinez, J. Hernandez-Garcia, and J. Lopez-Pavon, Non-unitarity, sterile neutrinos, and non-standard neutrino interactions, J. High Energy Phys. 04 (2017) 153. 